\title{
Novel cytological model for the identification of early oral cancer diagnostic markers: The carcinoma sequence model
}

\author{
MASAMI KAWAHARADA ${ }^{1,2}$, MANABU YAMAZAKI ${ }^{2}$, SATOSHI MARUYAMA $^{3}$, \\ TATSUYA ABÉ ${ }^{2}$, NYEIN NYEIN CHAN ${ }^{1,2}$, TAIICHI KITANO ${ }^{3}$, TADAHARU KOBAYASHI ${ }^{1}$, \\ TAKEYASU MAEDA ${ }^{4}$ and JUN-ICHI TANUMA ${ }^{2}$ \\ Divisions of ${ }^{1}$ Reconstructive Surgery for Oral and Maxillofacial Region and ${ }^{2}$ Oral Pathology, \\ Faculty of Dentistry and Graduate School of Medical and Dental Sciences, Niigata University, Chuo-ku, \\ Niigata 951-8514; ${ }^{3}$ Oral Pathology Section, Department of Surgical Pathology, Niigata University Hospital, \\ Chuo-ku, Niigata 951-8520; ${ }^{4}$ Research Center for Advanced Oral Science, Faculty of Dentistry and Graduate School of \\ Medical and Dental Sciences, Niigata University, Chuo-ku, Niigata 951-8514, Japan
}

Received October 7, 2021; Accepted December 29, 2021

DOI: 10.3892/ol.2022.13196

\begin{abstract}
Most oral squamous cell carcinomas (OSCCs) arise from a premalignant lesion, oral epithelial dysplasia; however, useful markers for the early detection of OSCC are lacking. The present study aimed to establish a novel experimental model to observe changes in the sequential expression patterns of mRNAs and proteins in a rat model of tongue cancer using liquid-based cytology techniques. Cytology specimens were collected at 2, 5, 8, 11, 14, 17 and 21 weeks from rats treated with 4-nitroquinoline 1-oxide to induce tongue cancer. The expression of candidate biomarkers was examined by performing immunocytochemistry and reverse transcription-quantitative PCR. The percentage of positively stained nuclei was calculated as the labeling index (LI). All rats developed OSCC of the tongue at 21 weeks. The mRNA expression levels of bromodomain protein 4 (Brd4), c-Myc and $T p 53$ were upregulated during the progression from negative for intraepithelial lesion or malignancy to squamous cell carcinoma (SCC). Brd4- and c-Myc-LI increased in low-grade squamous intraepithelial lesion, high-grade squamous intraepithelial lesion and SCC specimens. p53-LI was significantly increased in SCC specimens. This novel experimental model allowed the observation of sequential morphological changes and the expression patterns of mRNAs and proteins during carcinogenesis. Combining immunocytochemistry
\end{abstract}

Correspondence to: Professor Jun-Ichi Tanuma, Division of Oral Pathology, Faculty of Dentistry and Graduate School of Medical and Dental Sciences, Niigata University, 2-5274 Gakkocho-dori, Chuo-ku, Niigata 951-8514, Japan

E-mail: tanuma@dent.niigata-u.ac.jp

Key words: oral squamous cell carcinoma, oral cancer, bromodomain protein 4, c-Myc, p53, cytology, liquid-based cytology, immunocytochemistry, 4-nitroquinoline 1-oxide with cytology-based diagnoses may potentially improve the diagnostic accuracy of OSCC.

\section{Introduction}

The global incidence of oral squamous cell carcinoma (OSCC) has increased to more than 355,000 new patients and 177,000 related deaths annually (1-3). The most common sites for intraoral cancer are the tongue, gingiva, and floor of the mouth, which account for more than half of all OSCCs (1). The oral cavity is central to several essential functions such as speech, breathing, and feeding, and cancers in this region can severely affect several functions essential for day-to-day life (4). Early detection and treatment of OSCC can improve quality of life. Most OSCCs arise from oral epithelial dysplasia (OED). Several studies have focused on OSCC detection; however, candidate biomarkers for the early detection of malignant transformation have not yet been identified (5). This may be attributed to the lack of an experimental model that allows continuous observation of molecular changes occurring in the dysplasia-carcinoma sequence in the same organism (6).

Liquid-based cytology (LBC) is a non-invasive technique that can be used for the early diagnosis of OSCC. LBC has higher diagnostic accuracy than conventional cytology; moreover, gene expression analysis and immunocytochemistry (ICC) can be performed on the residual LBC specimens (7-11). There are several reports on ICC and gene expression analysis; however, none of the studies have reported the continuous changes in morphology and the gene and protein expression during carcinogenesis. Most cytology specimens are collected from superficial or keratinized cell layers, and the changes occurring in this layer during carcinogenesis need to be observed. Therefore, the focus should be on identifying biomarkers expressed in the keratinized cell layers.

Animal experiments are useful for observing changes in molecular expression during carcinogenesis. We previously reported that Dark-Agouti (DA) rats are highly susceptible to 4-nitroquinoline 1-oxide (4NQO)-induced tongue 
cancer (TC) (12-14). DA rats with 4NQO-induced TC are considered an ideal experimental model for human TC because tumors in both share several morphological, molecular, and biological properties (14). Using LBC techniques on this model, it is theoretically possible to continuously observe malignant transformation events in the same organism. Previous studies analyzed quantitative trait loci, loss of heterozygosity, and microarray results in 4NQO-induced TC and identified that genes encoding c-Myc and p53 are associated with the promotion and progression of OSCC $(12,14-16)$. Therefore, we focused on these genes in this study. Furthermore, we analyzed the role of bromodomain protein 4 (Brd4) because Brd4 transcriptionally regulates Myc expression and is expressed in the superficial or keratinized cell layers during the early stages of carcinogenesis $(17,18)$.

Brd4 is a member of the bromodomain and extraterminal domain (BET) family of transcriptional regulatory proteins. It plays a key role in carcinogenesis and cancer progression (19). Brd4 expression increases during 4NQO-induced carcinogenesis in animal models, and it modulates tumorigenicity and tumor overgrowth (18). Significantly higher levels of Brd4 mRNA and protein expression are observed in the head and neck squamous cell carcinoma (SCC) tissues, compared to that in normal tissues $(18,20,21)$. Brd4 expression correlates with matrix metallopeptidase 2 expression in OSCC. Reportedly, $\mathrm{Brd} 4$ is overexpressed in OSCC patients with lymph node metastasis than in patients without metastasis $(22,23)$. Furthermore, Brd4 binds and activates the c-Myc promoter to induce the c-Myc overexpression (24). c-Myc plays a key role in cell proliferation, metabolism, differentiation, promotion of p53 expression, and apoptosis. However, when Tp53 is mutated, cell death is avoided, leading to the development of OSCC $(25,26)$. Reportedly, c-Myc expression levels are associated with the advanced stages of OED and OSCC $(18,27,28)$, wherein c-Myc and p53 are overexpressed in the early stages of oral carcinogenesis. Therefore, these are considered useful markers for the early detection of premalignant lesions using immunohistochemistry (IHC) (29). However, none of the studies have explored the usefulness and reliability of these markers for ICC-based cancer detection.

The aim of this study was to establish an experimental model, wherein the sequential mRNA and protein expression patterns of Brd4, c-Myc, and p53 could be observed in the same organism using $\mathrm{LBC}$ in a $4 \mathrm{NQO}$-induced rat model of TC and verify whether these biomarkers are useful for the early detection of OSCC when combined with cytological diagnosis.

\section{Materials and methods}

Animals. Inbred DA rats (DA/Slc) were purchased from the Shizuoka Laboratory Animal Center (Hamamatsu, Japan). All rats were housed in a controlled environment with a $12 \mathrm{~h}$ light/dark cycle and temperature of $22 \pm 2^{\circ} \mathrm{C}$ and fed a commercial pellet-based feed (Nosan, Yokohama, Japan). Fifty-one 4-weeks old male DA rats were used for the cytological analysis, and twelve rats were used for histological experiments. This study was reviewed by the Committee of the Ethics on Animal Experiments in Niigata University and carried out according to the Guidelines for Animal Experiments in
Niigata University, Niigata, Japan (SA00507). All experiments were conducted in accordance with relevant national legislation on the use of animals for research.

TC induction. A stock solution of 4NQO (200 mg/l in 5\% ethanol; Nacalai Tesque Inc., Kyoto, Japan) was prepared and stored at $4^{\circ} \mathrm{C}$ until use. Starting 6 weeks of age, all rats were given drinking water containing $0.001 \%$ 4NQO ad libitum. At week 21 of the experiment, all rats were sacrificed by sodium pentobarbital (150 mg/kg body wt, i.p.), followed by full necropsy and histopathological examinations.

LBC specimens. LBC specimens were collected from the tongue region of rats at $2,5,8,11,14,17$, and 21 weeks of the experiment. An Orcellex brush (Rovers Medical Devices B.V., Oss, Netherlands) or interdental brush (Dentalpro Co., Osaka, Japan) was rotated on the lesion surface 20 times. The collected contents were transferred into a methanol-based preservative solution (SurePath, BD Diagnostics, Franklin Lakes, NJ, USA) or RNAlater reagent (Ambion, Austin, TX, USA). LBC preparations were processed according to the manufacturer's protocol (30). Fixed specimens were rehydrated with distilled water and subsequently, subjected to nuclear staining with hematoxylin solution and cytoplasmic staining with Orange G solution (Muto Pure Chemicals Co., Ltd, Tokyo, Japan) and Eosin Azure solution (Muto Pure Chemicals Co., Ltd.). Cytological diagnosis was based on the oral Bethesda system (31).

Histopathological specimens. We performed another carcinogenesis experiment using $12 \mathrm{DA}$ rats; three rats without $4 \mathrm{NQO}$ treatment were analyzed as controls. We sacrificed three rats each (a total of nine DA rats) at the time point of the low-grade squamous intraepithelial lesion (LSIL), high-grade squamous intraepithelial lesion (HSIL), and SCC formation. The tongue specimens were fixed in buffered $10 \%$ formalin and embedded in paraffin. Serial 4- $\mu \mathrm{m}$ sections were cut, dewaxed in xylene, and rehydrated in graded ethanol. The normal mucosal epithelium, hyperplasia, OED, and SCC sections were stained with hematoxylin and eosin to confirm the histological diagnosis, while the other sections were used for immunohistochemical analyses.

Immunostaining of Brd4, c-Myc, and p53 in the cytological and the histological specimens. Immunostaining was performed to observe the expression patterns of $\mathrm{Brd} 4$, c-Myc, and p53 in the cytological and histological specimens. All slides were subjected to antigen retrieval in a microwave oven, with a maximum strength of $1000 \mathrm{~W}$, using EDTA ( $\mathrm{pH} 8.0$ ), for $20 \mathrm{~min}$ followed by incubation with either a rabbit polyclonal anti-p53 antibody (1:50 dilution at room temperature (RT, $24 \pm 2^{\circ} \mathrm{C}$ ) for $2 \mathrm{~h}$; clone ab131442; Abcam, Cambridge, MA, USA), a rabbit monoclonal anti-c-Myc antibody (1:100 dilution at RT for $2 \mathrm{~h}$; clone ab32072; Abcam), or a rabbit monoclonal anti-BRD4 antibody (1:100 dilution at RT for 2 h; clone ab128874; Abcam). The slides were then washed and incubated with the Envision+/HRP system (Dako, Glostrup, Denmark). Immunoreactive cells were visualized using DAB (Dojindo, Kumamoto, Japan) and counterstained with hematoxylin. 
Table I. Cytological diagnoses of 51 rats at each week.

4NQO treatment period (weeks)

\begin{tabular}{|c|c|c|c|c|c|c|c|}
\hline Cytological diagnosis & 2 & 5 & 8 & 11 & 14 & 17 & 21 \\
\hline NILM & $51(100.0)$ & $51(100.0)$ & $51(100.0)$ & $44(86.3)$ & 25 (49.0) & $7(13.7)$ & $0(0.0)$ \\
\hline LSIL & $0(0.0)$ & $0(0.0)$ & $0(0.0)$ & $7(13.7)$ & $25(49.0)$ & $24(47.1)$ & $0(0.0)$ \\
\hline HSIL & $0(0.0)$ & $0(0.0)$ & $0(0.0)$ & $0(0.0)$ & $1(2.0)$ & $18(35.3)$ & $0(0.0)$ \\
\hline SCC & $0(0.0)$ & $0(0.0)$ & $0(0.0)$ & $0(0.0)$ & $0(0.0)$ & $2(3.9)$ & $51(100.0)$ \\
\hline
\end{tabular}

Data are presented as the number of rats (\%). 4NQO, 4-nitroquinoline 1-oxide; NILM, negative for intraepithelial lesion or malignancy; LSIL, low-grade squamous intraepithelial lesion; HSIL, high-grade squamous intraepithelial lesion; SCC, squamous cell carcinoma.

Labeling index analysis of ICC. The percentages of positively stained nuclei in specimens stained with each of the antibodies (anti-Brd4, anti-c-Myc, and anti-p53) were calculated as the labeling index (LI) using the images captured at x200 magnification and analyzed using e-Count 2 cell counting software (e-Path, Kanagawa, Japan). Six random fields containing an average of 200 cells each were selected for analysis, and the average value for LI was determined.

RNA isolation and quantitative real-time PCR ( $q R T-P C R)$. The LBC specimens in the RNAlater reagent were processed using the NucleoSpin RNA XS-kit (Macherey-Nagel, Düren, Germany) according to the manufacturer's instructions. Reverse transcription was carried out using the High-Capacity cDNA Reverse Transcription Kit with RNase inhibitor (Applied Biosystems, Foster City, CA, USA) according to the manufacturer's protocol. cDNA was amplified using a TaqMan Universal PCR Master Mix (Applied Biosystems) and TaqMan Gene Expression Assays (Applied Biosystems) for Brd4 (Rn01535560_m1), Myc (Rn07310910_m1), and Tp53 (Rn00755717_m1); 18S mRNA (Hs99999901_s1) was used as the internal standard (32). To compare the levels of target gene transcripts in LSIL, HSIL, and SCC samples with those in negative for intraepithelial lesion or malignancy (NILM) samples, the $2^{-\Delta \Delta \mathrm{Cq}}$ method was used to calculate relative mRNA levels (33).

Statistical analyses. The Shapiro-Wilk tests showed that the Brd4, c-Myc, and p53-LI and the Brd4 and Tp53 mRNA levels were normally distributed; therefore, these data were analyzed parametrically. We performed ANOVA with a post-hoc Tukey test for comparisons between multiple groups. In contrast, the c-Myc mRNA levels were not normally distributed; therefore, the data were assessed with a Kruskal-Wallis followed by a Dunn's post hoc test. The correlation between mRNA expression and LI was assessed using the Spearman rank correlation test. We evaluated the diagnostic ability of these biomarkers using receiver operating characteristic (ROC) curves. As a global measure for the accuracy of diagnosis, we also calculated the area under the ROC curve (AUC) for each biomarker. The optimal cut-off values for the LIs for Brd4, c-Myc, and p53 were determined using 'closest-topleft' (34). In addition, sample size calculations showed that 12 samples were necessary for each group to reach $80 \%$ power at a $5 \%$ significance level. All statistical comparisons were performed using GraphPad Prism for Windows version 6.00 (GraphPad Software Inc., San Diego, CA, USA) and R version 4.0.2 (R Foundation, Vienna, Austria). A p-value of $<0.05$ was considered significant, and all statistical tests were two-sided.

\section{Results}

Sample collection. The cytology specimens from the tongues of 51 rats were classified into four groups, NILM, LSIL, HSIL, and SCC, according to the oral Bethesda system. LSIL was recognized after 11 weeks of treatment with 4NQO. At 17 weeks, all the diagnostic classifications, from NILM to SCC, could be recognized. Finally, all the samples were evaluated as SCC at 21 weeks. The results of the cytopathological examination are summarized in Table I. Furthermore, the mean incidence periods of LSIL, HSIL, and SCC were $14.3 \pm 2.1,16.8 \pm 0.7$, and $20.8 \pm 0.8$ weeks after the beginning of the carcinogenesis experiments, respectively.

Expression analysis of Brd4, $c-M y c$, and p53 using ICC and IHC. Following Papanicolaou staining, NILM specimens showed orangeophilic keratinized cells without atypical or higher brightness (Fig. 1A). The LSIL specimens had mild atypical high-bright orangeophilic keratinized cells (Fig. 1B), whereas the HSIL specimens had moderate atypical high-bright orangeophilic keratinized cells (Fig. 1C). In addition, several atypical parabasal/basal cell clusters were detected in SCC specimens (Fig. 1D). NILM, LSIL, HSIL, and SCC were further evaluated by performing ICC staining for Brd4, c-Myc, and p53. NILM specimens tested mostly negative for these markers (Fig. 1E, I and M). In LSIL and HSIL specimens, positive staining for Brd4 and c-Myc was observed along with the atypically enlarged nuclei of the superficial and intermediate cells (Fig. 1F, G, J and K). In SCC specimens, Brd4 and c-Myc expression were detected in the parabasal/basal cells that appeared small and rounded and showed clear evidence of nuclear changes, such as nuclear enlargement, nuclear shape abnormalities, and increased nuclear to cytoplasmic ratios (Fig. $1 \mathrm{H}$ and L). In contrast, p53 expression was observed only in SCC specimens, localized in the atypical nuclei of parabasal/basal cells (Fig. 1N-P). 


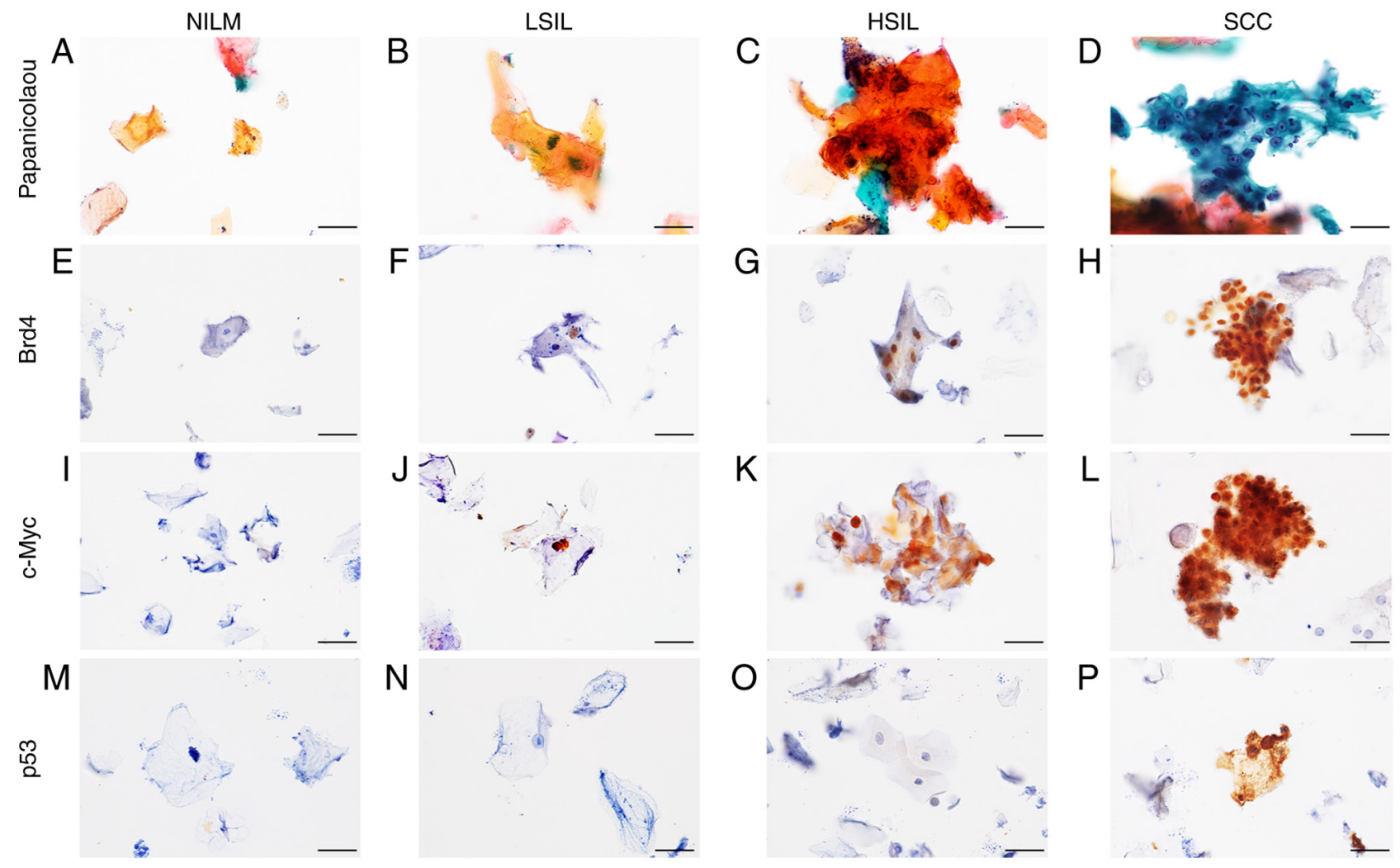

Figure 1. Immunoexpression of Brd4, c-Myc and p53 in cytological specimens. Representative cytological findings based on Papanicolaou staining of liquid-based cytology specimens: (A) NILM, (B) LSIL, (C) HSIL and (D) SCC. (E-H) Brd4 immunocytochemical staining of oral smears. Although Brd4 staining was generally negative in (E) NILM specimens, (F) positive staining in the nuclei was observed in LSIL, (G) HSIL and (H) SCC specimens. (I-L) c-Myc immunocytochemical staining of oral smears. Although c-Myc staining was generally negative in (I) NILM specimens, positive staining in the nuclei was observed in (J) LSIL, (K) HSIL and (L) SCC specimens. (M-P) p53 immunocytochemical staining of oral smears. Although p53 staining was generally negative in (M) NILM, (N) LSIL and (O) HSIL specimens, positive staining in the nuclei was observed in (P) SCC specimens. Original magnification, x600. Scale bars, $20 \mu \mathrm{m}$. NILM, negative for intraepithelial lesion or malignancy; LSIL, low-grade squamous intraepithelial lesion; HSIL, high-grade squamous intraepithelial lesion; SCC, squamous cell carcinoma; Brd4, bromodomain protein 4.

After confirming the histological diagnosis of the normal mucosal epithelium (Fig. 2A), hyperplasia (Fig. 2B), OED (Fig. 2C), and SCC (Fig. 2D) by hematoxylin and eosin staining, the expression of Brd4, c-Myc, and p53 was further compared by IHC staining. Brd4 expression was observed from the basal layer to the superficial layer in the hyperplasia, OED, and OSCC tissue specimens (Fig. 2E-H), while the c-Myc expression was limited to the basal and parabasal layers of the hyperplasia. c-Myc positive cells were observed from the basal layer to the superficial layers in the OED and OSCC tissue specimens (Fig. 2I-L). Diffuse and intensive p53 nuclear positivity was detected in OSCC tissue specimens but not overexpression was detected in the normal mucosal epithelium, hyperplasia, and OED tissue specimens (Fig. 2M-P). Thus, consistent expression patterns were observed using ICC and IHC.

mRNA expression levels of candidate markers in LBC specimens. Results of qRT-PCR for Brd4, c-Myc, and Tp53 mRNA expression revealed a statistically significant difference in the expression of each marker among the oral Bethesda categories $(\mathrm{P}<0.01$; Tukey's multiple comparisons test for $\mathrm{Brd} 4$ and Tp53, Kruskal-Wallis test for c-Myc). In addition, the levels of Brd4, c-Myc, and Tp53 mRNAs were significantly higher in SCC specimens than that in the NILM, LSIL, and HSIL specimens $(\mathrm{P}<0.01$; Fig. $3 \mathrm{~A}-\mathrm{C})$. These results showed that expression levels of the Brd4, c-Myc, and Tp53 mRNAs were upregulated during the carcinogenesis in the 4NQO-induced TC model.

Protein levels of candidate markers in LBC specimens. To identify Brd4, c-Myc, and p53 protein expression patterns in the different grades within the oral Bethesda system, we analyzed the expression of Brd4, c-Myc, and p53 proteins in NILM, LSIL, HSIL, and SCC specimens using ICC. Tukey's multiple comparisons test showed significant differences in the LI for Brd4 (BRD4-LI), c-Myc (c-Myc-LI), and p53 (p53-LI) among the oral Bethesda categories $(\mathrm{P}<0.01)$. The BRD4-LI was significantly higher in HSIL and SCC specimens than that in the NILM and LSIL specimens, based on the ICC results $(\mathrm{P}<0.01$; Tukey's multiple comparisons test) (Fig. 3D). In line with Brd4 expression, c-Myc-LI was significantly higher in the HSIL and SCC specimens than that in the NILM and LSIL specimens ( $\mathrm{P}<0.01$; Tukey's multiple comparisons test) (Fig. 3E). Similarly, p53-LI was significantly higher in the SCC specimens compared to that in the NILM, LSIL, and HSIL specimens ( $\mathrm{P}<0.01$; Tukey's multiple comparisons test) (Fig. 3F). These results showed that the expression of Brd4, c-Myc, and p53 proteins increased with disease severity. In particular, the levels of Brd4 and c-Myc were higher in the LSIL and HSIL specimens. 


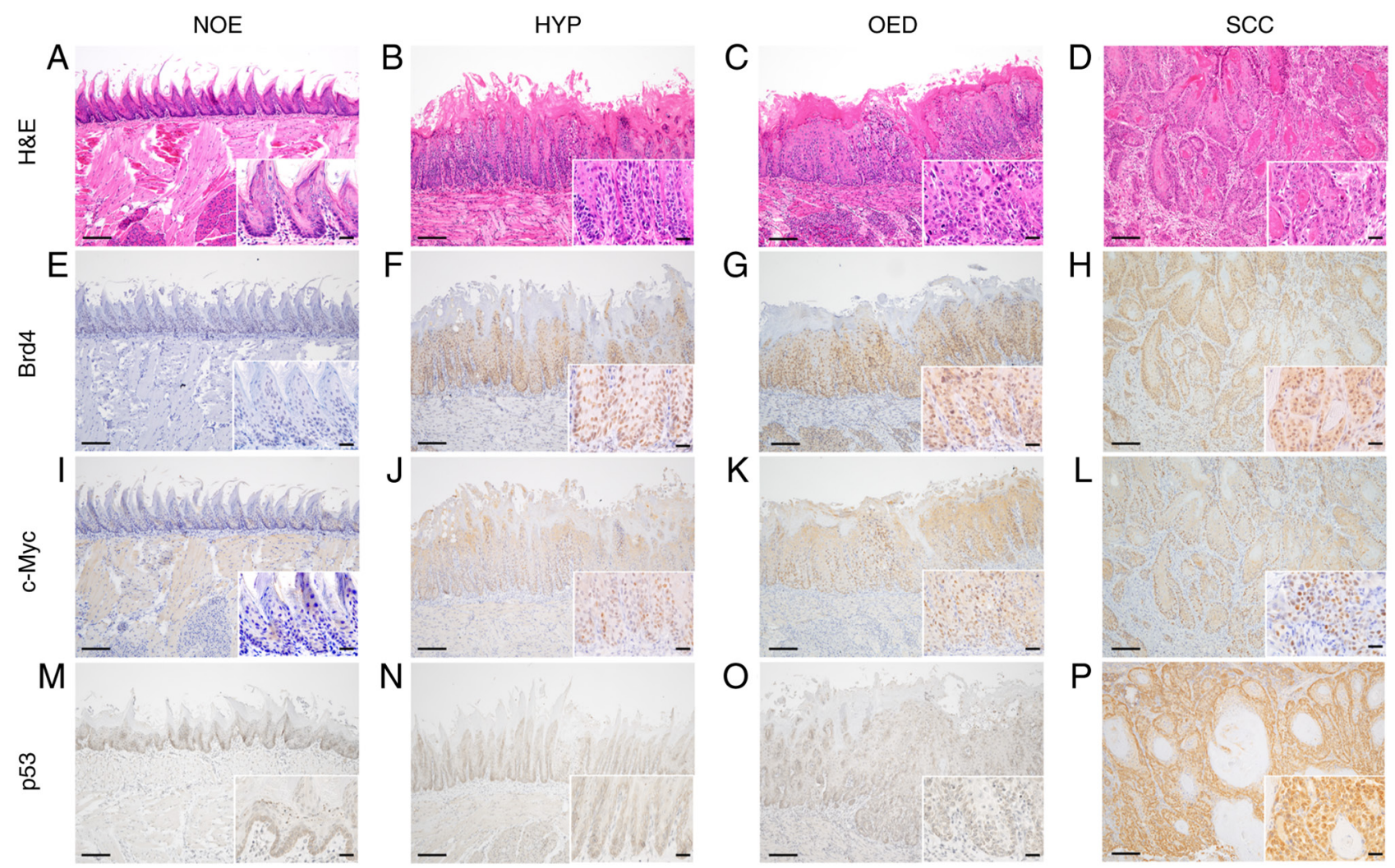

Figure 2. Representative histopathological and immunohistochemical findings for Brd4, c-Myc and $\mathrm{p} 53$ expression patterns in control and 4NQO-treated rats at 21 weeks. (A, E, I, M) NOE, (B, F, J, N) HYP, (C, G, K, O) OED and (D, H, L, P) SCC. (A-D) H\&E, (E-H) Brd4, (I-L) c-Myc and (M-P) p53. Original magnification, x100 and 400 (inset). Scale bars, 100 and $20 \mu \mathrm{m}$ (inset). 4NQO, 4-nitroquinoline 1-oxide; NOE, normal epithelium; HYP, hyperplasia; OED, oral epithelial dysplasia; SCC, squamous cell carcinoma; H\&E, hematoxylin and eosin; Brd4, bromodomain protein 4.

Relationship between mRNA expression levels and LI for $B r d 4, c-M y c$, and $p 53$. To analyze the relationship between mRNA expression levels and the LI for ICC, we performed the correlation analysis for each candidate marker. Fig. 4 shows the relationship between Brd4, c-Myc, and p53 mRNA expression levels. Significant correlations were detected for $\mathrm{Brd} 4$ ( $\mathrm{R}=0.761, \mathrm{P}<0.01$; Fig. 4A), c-Myc $(\mathrm{R}=0.794, \mathrm{P}<0.01$; Fig. 4B) and p53 ( $\mathrm{R}=0.599 ; \mathrm{P}<0.01$; Fig. $4 \mathrm{C})$ expression levels.

Diagnostic accuracy of candidate markers. To evaluate the diagnostic accuracy of the increased LI for individual candidate markers, we stratified the LI of each marker as positive (LI below cut-off) or negative (LI above the cut-off). We calculated the sensitivities and specificities to detect LSIL or a higher category by performing ROC analysis (Fig. 5). The AUCs were 0.833 (standard error [SE]: 0.039; 95\% CI: 0.758-0.909) for Brd4, 0.849 (SE: 0.036; 95\% CI: 0.778-0.921) for c-Myc, and 0.829 (SE: 0.042; 95\% CI: 0.747-0.911) for p53. When we classified into negative (NILM) and positive (LSIL, HSIL, and SCC), Table II shows the sensitivity, specificity, false-negative rate (FNR), negative predictive value (NPV), positive predictive value (PPV), and diagnostic accuracy of all markers. Using the oral Bethesda system, cut-off BRD4-LI values greater than 6.0\% were observed in $60.0 \%$ of LSIL and $100 \%$ of HSIL specimens; likewise, for c-Myc, cut-off c-Myc-LI values greater than $12.0 \%$ were observed in $50.0 \%$ of LSIL and $78.0 \%$ of HSIL specimens. In contrast, a cut-off p53-LI greater than $3.6 \%$ was observed in only $20 \%$ of LSIL and $58 \%$ of HSIL specimens.

\section{Discussion}

To identify biomarkers useful for the early diagnosis of OSCC, we established a novel experimental rat model that would enable evaluating the biological changes occurring during the multi-step carcinogenesis process in the same animal. In this result, Papanicolaou smears showed sequential progression from NILM to LSIL/HSIL to SCC, and the morphological changes were similar to that observed during the progression of human oral carcinogenesis (35). We also succeeded, for the first time, in performing ICC and qRT-PCR on mRNA isolated from cytology specimens in a 4NQO-induced rat model of TC. Several studies have suggested that the 4NQO-induced rat model of TC can be used to visualize histological as well as molecular changes in human oral carcinogenesis $(14,36,37)$. In addition, Arduino et al (38) reported that the mean age of the patient diagnosed with $\mathrm{OED}$ is 63.8 years ( $\mathrm{SD} \pm 10.7$ years), and the mean period for malignant transformation of OED is 29.8 months (range: 9-120 months). These results suggest that in humans, the time for OED to develop (approximately 60 years) is longer with a short period of transformation from OED to OSCC, and are consistent with our results in the rat model. Taken together, these findings support that our 4NQO-induced rat model of TC may be a suitable model of human OSCC. Furthermore, to the best of our knowledge, the morphological and molecular changes using cytology specimens have not been explored in previous studies. Therefore, we believe this is the first study to demonstrate the changes occurring in morphology 
A
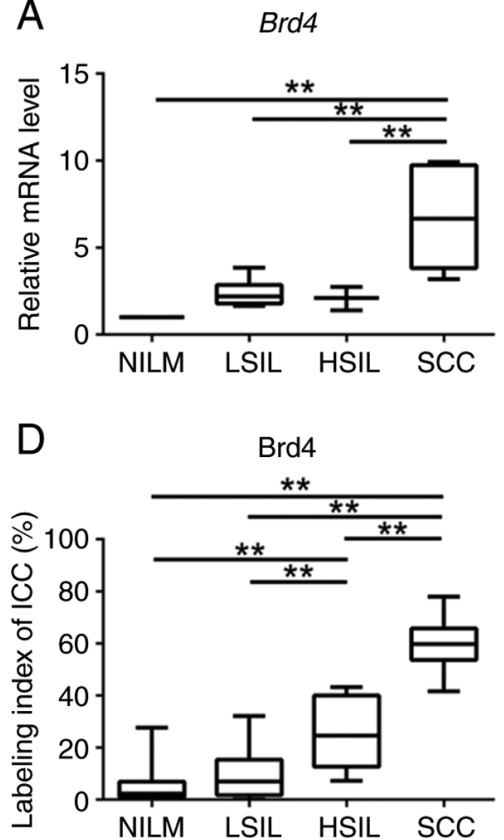

B

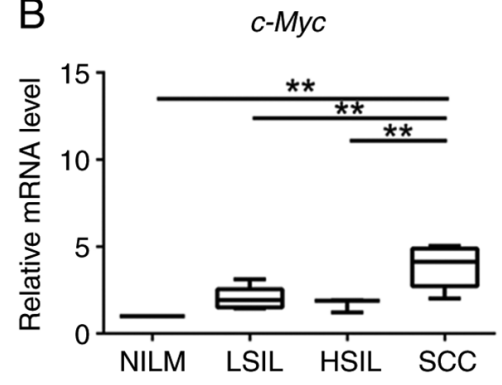

$\mathrm{E}$

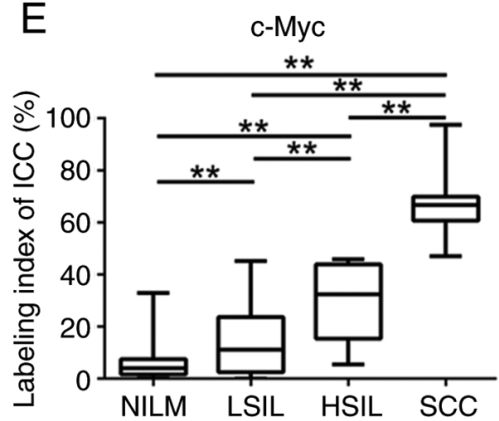

C

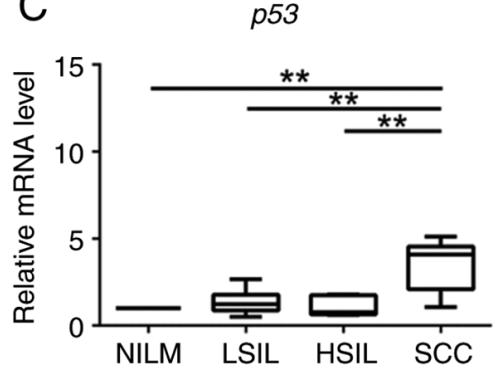

$\mathrm{F}$

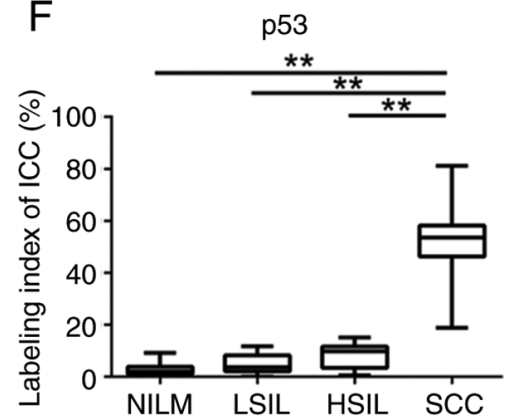

Figure 3. Box plots showing normalized expression of (A) Brd4, (B) c-Myc and (C) p53 in NILM, LSIL, HSIL and SCC specimens. The box represents 50\% quartiles $(>25 \%$ and $<75 \%$ ), and the solid line within each box is the median gene expression value. ANOVA followed by Tukey's multiple comparisons test and the Kruskal-Wallis test followed by Dunn's post hoc test were used to determine statistical significance. Boxplots for labeling indices from ICC of (D) Brd4, (E) c-Myc and (F) p53 in NILM, LSIL, HSIL and SCC specimens. ANOVA followed by Tukey's multiple comparisons test was used to determine statistical significance. ${ }^{* *} \mathrm{P}<0.01$, as indicated. NILM, negative for intraepithelial lesion or malignancy; LSIL, low-grade squamous intraepithelial lesion; HSIL, high-grade squamous intraepithelial lesion; SCC, squamous cell carcinoma; ICC, immunocytochemistry; Brd4, bromodomain protein 4.

A

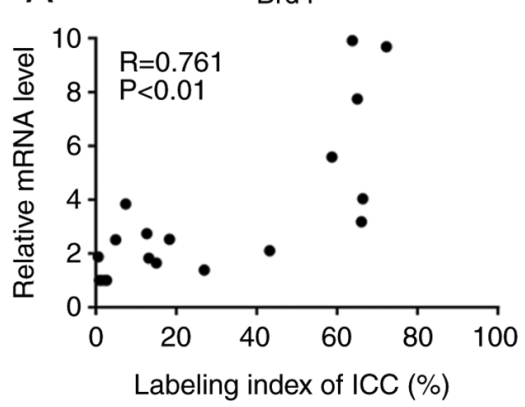

B

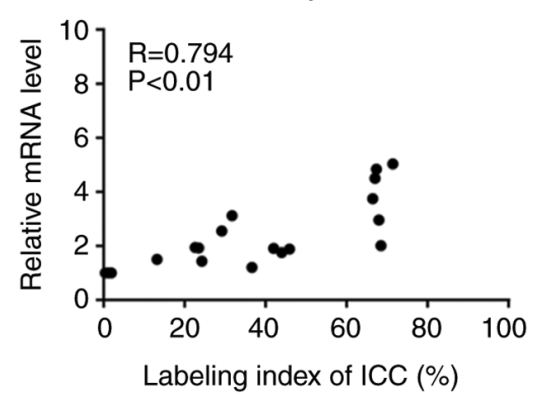

C

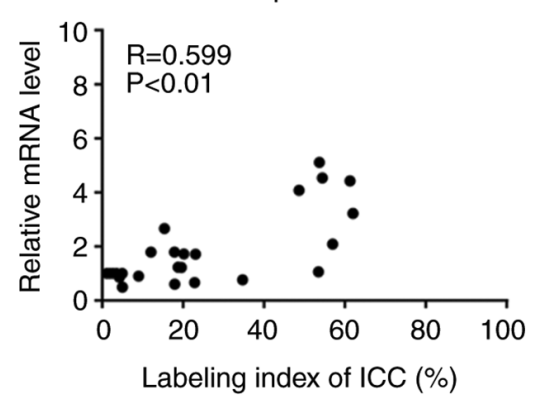

Figure 4. Correlation between labeling index and the relative mRNA level of each marker (Brd4, c-Myc, p53) in cytological specimens. These markers displayed significant positive correlations: (A) Brd4 ( $\mathrm{R}=0.761, \mathrm{P}<0.01)$, (B) c-Myc $(\mathrm{R}=0.794, \mathrm{P}<0.01)$ and $(\mathrm{C}) \mathrm{p} 53$ ( $\mathrm{R}=0.599$, $\mathrm{P}<0.01)$. ICC, immunocytochemistry; Brd4, bromodomain protein 4.

and mRNA and protein expression during carcinogenesis, using the cytology specimens obtained from the same organism.

As most of the cytological specimens are collected from the superficial and keratinized cell layers, the biomarkers expressed in these layers could be useful. A previous study has shown that immunohistochemical staining for cytokeratin 13 (CK13), CK17, and p53 is useful to adjunct the histological diagnosis of OED and OSCC. However, the expression patterns of CK13 and CK17 vary in the reactive lesions and are not specific for the neoplastic changes (39). Similar results have also been reported in other studies. For example, Noda et al (40) investigated the immunocytochemical expression patterns of CK13 and CK17 and reported that these markers could not distinguish non-neoplastic and neoplastic lesions in the oral cytology specimens. In this study, we selected p53 as a candidate marker because p53 overexpression is reportedly involved in OSCC development, and Tp53 mutations are observed in OED and OSCC (41). However, in this study, the immunohistochemical results revealed the absence of p53 overexpression in the normal mucosal epithelium, hyperplasia, and OED specimens; therefore, it was difficult to detect the overexpression of p53 in the cytology specimens from the premalignant lesions. In contrast, hyperplasia, OED, and OSCC specimens showed Brd4 expression from the basal to superficial layers. c-Myc was expressed in the basal to spinous cell layer in the hyperplasia specimens and in the basal to superficial layer in the OED and SCC specimens; therefore, we considered Brd4, and c-Myc as candidate markers that were detected by ICC in the hyperplasia and OED specimens and examined their usefulness and reliability in this novel experimental model. 
Table II. Cut-off values, sensitivity, specificity, FNRs, NPVs and PPVs for BRD4-LI, c-Myc-LI and p53-LI.

\begin{tabular}{lccccccc}
\hline Markers & Cut-off value, $\%$ & Sensitivity, $\%$ & Specificity, $\%$ & FNR, $\%$ & PPV, $\%$ & NPV, $\%$ & Accuracy, $\%$ \\
\hline Brd4 & 6.0 & 81.5 & 73.9 & 18.5 & 81.5 & 73.9 & 78.3 \\
c-Myc & 12.0 & 74.6 & 87.0 & 25.3 & 89.3 & 70.2 & 79.6 \\
p53 & 3.6 & 59.0 & 100.0 & 41.0 & 100.0 & 54.4 & 72.5
\end{tabular}

Brd4, bromodomain protein 4; FNR, false negative ratio; PPV, positive predictive value; NPV, negative predictive value; LI, labeling index.

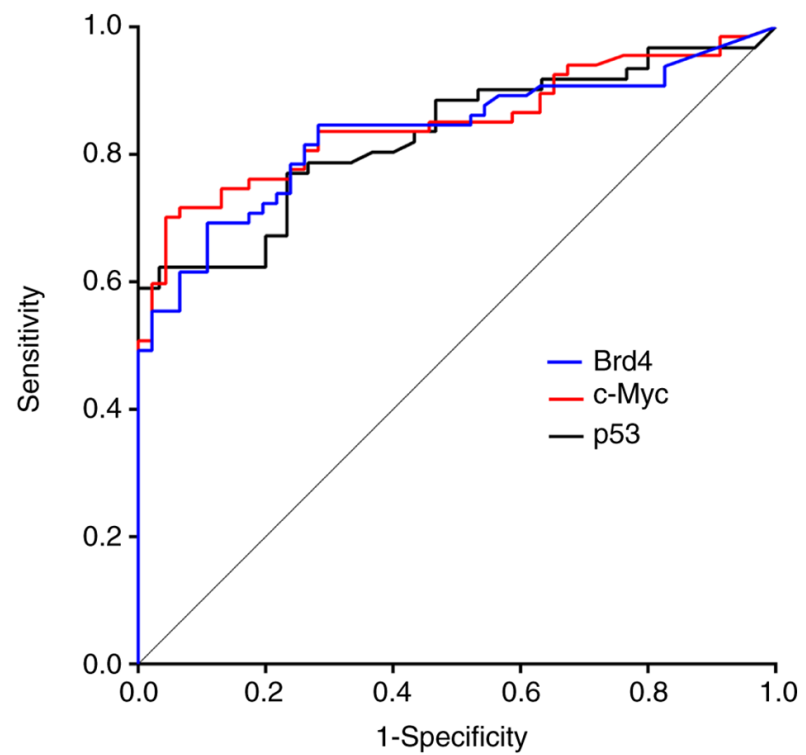

Figure 5. Receiver operating characteristic analysis for low-grade squamous intraepithelial lesion or higher category specimens screening using Brd4 (blue line), c-Myc (red line) and p53 (black line) as candidate markers. The optimal cut-off values of each marker were calculated using 'closest-topleft' (34). Brd4, bromodomain protein 4.

Results of the qRT-PCR analysis showed that Brd4, c-Myc, and Tp53 mRNA expression increased with the progression from NILM to SCC, and each marker was significantly overexpressed in SCC specimens than in the NILM, LSIL, and HSIL specimens. These observations are consistent with those reported in the previous studies in OSCC $(18,42,43)$. The level of Brd4 mRNA is aberrantly upregulated in head and neck SCC samples and a 4NQO-induced animal model of OSCC (18). c-Myc and p53 are overexpressed in OSCC tissues and TSCCA and CAL-27 cell lines than in normal tissues and normal human oral keratinocyte cells $(37,42)$.

The LI values for Brd4, c-Myc, and p53 increased with the progression from NILM to SCC. Although c-Myc immunostaining patterns can be divided into three types-nuclear, granular perinuclear, and diffuse cytoplasmic-we analyzed only the nuclear-positive patterns. In frozen specimens of normal tissues, Loke et al (44) found a predominantly nuclear localization of the c-Myc protein in the liver, spleen, kidney, lungs, and so on; however, when tissues were fixed in formalin, this pattern was altered, suggesting possible protein translocation to the cytoplasm during fixation. c-Myc is ubiquitinated in the nucleus and exported to the cytoplasm for degradation (45). $\mathrm{c}-\mathrm{Myc}$ is a nuclear transcription factor, and therefore, it is most active in the nucleus (46). The c-Myc expression pattern in OSCC remains debatable; however, collectively, these studies indicate that the cytoplasmic localization of c-Myc immunoreactivity is unlikely to be considered positive, and it may be reasonable to evaluate the nuclear localization of c-Myc in OSCC. Moreover, the methods used for calculating LI were adequate because a significant correlation was observed between the LI and mRNA expression level for each marker (Fig. 4). Also, ICC was more effective in detecting positive cells than qRT-PCR because ICC calculates the LI for the hot spots in the cytology specimens, whereas qRT-PCR evaluates entire specimens. Consequently, ICC is a more useful method than qRT-PCR for the early detection of OSCC from cytology specimens.

LI for Brd4 and c-Myc increased significantly during the early stages of the carcinogenesis, and therefore, they may be good predictors of OSCC. Cytologic diagnoses based on morphologic changes are frequently difficult because of the occurrence of false negatives. In particular, differentiating between reactive/regenerative or neoplastic changes, namely NILM and LSIL, is challenging (40). Our analysis showed that the cells positive for Brd4 or c-Myc expression were more frequently detected in LSIL specimens than the cells positive for p53 expression. Therefore, assessing BRD4-LI and c-Myc-LI by ICC, in addition to cytological diagnosis, can improve the diagnostic accuracy of the cytology of OSCC. c-Myc is a well-known oncogene and a downstream target of Brd4 (18). c-Myc promotes p53 expression; it also induces the expression of cyclins $\mathrm{D} / \mathrm{E}$ and cyclin-dependent protein kinases 2/4/6 and represses $\mathrm{p} 21^{\mathrm{CIP} 1}$ and $\mathrm{p} 27^{\mathrm{KIP} 1}$ levels, leading to cell cycle progression (47-52). Brd4 and c-Myc expressions are usually upregulated in OSCC and significantly associated with aggressive clinicopathological features and poor survival $(18,53)$. However, their usefulness as markers for the early detection of OSCC remains unknown. Combining cytology and ICC to increase diagnostic accuracy is widely reported for other cancers, but just a few studies have been conducted for evaluating the pathological conditions of the oral cavity $(8,54-59)$. Therefore, this study offers a new cytological diagnosis tool for OSCC.

In conclusion, the novel experimental model reported in this study allowed us to observe the sequential morphologic changes and expression patterns of mRNAs and proteins in the same animal during carcinogenesis. Our data suggested that ICC-based detection of Brd4 or c-Myc expression from cytology specimens could improve the diagnostic accuracy. Therefore, in combination with cytology, immunocytochemistry can improve the accuracy of OSCC diagnosis. 


\section{Acknowledgements}

Not applicable.

\section{Funding}

This research was funded by the Grants-in-Aid for Scientific Research from the Japan Society for the Promotion of Science (JSPS KAKENHI grant no. 19K10069).

\section{Availability of data and materials}

The datasets used and/or analyzed during the current study are available from the corresponding author on reasonable request.

\section{Authors' contributions}

MK was responsible for data acquisition (management of patient samples), analysis and interpretation, development of methodology, and the writing, editing/reviewing and intellectual content of the manuscript. MY designed the methodology, and was responsible for the writing, editing/reviewing and intellectual content of the manuscript. SM was responsible for the analysis and interpretation of data, and the writing, editing/reviewing and intellectual content of the manuscript. TA, TKo and TM were responsible for the analysis and interpretation of data, and the writing, editing/reviewing and intellectual content of the manuscript. NC and TKi were responsible for acquisition of data (management of patient samples). JT was responsible for the study concept and design, development of methodology, analysis and interpretation of data, writing, editing/reviewing and intellectual content of the manuscript, and study supervision. MK and JT confirm the authenticity of all the raw data. All authors have read and approved the final manuscript.

\section{Ethics approval and consent to participate}

This study was approved by the Animal Care Guidelines of Niigata University (Niigata, Japan; approval no. SA00507).

\section{Patient consent for publication}

Not applicable.

\section{Competing interests}

The authors declare that they have no competing interests.

\section{References}

1. El-Naggar AK, Chan JKC, Grandis JR, Takata T, Slootweg PJ (eds): World Health Organization classification of Head and Neck Tumours. Vol 9. 4th edition. IARC Press, Lyon, pp 109-111, 2017.

2. Bray F, Ferlay J, Soerjomataram I, Siegel RL, Torre LA and Jemal A: Global cancer statistics 2018: GLOBOCAN estimates of incidence and mortality worldwide for 36 cancers in 185 countries. CA Cancer J Clin 68: 394-424, 2018.

3. Miranda-Filho A and Bray F: Global patterns and trends in cancers of the lip, tongue and mouth. Oral Oncol 102: 104551, 2020 .
4. Tirelli G, Gatto A, Bonini P, Tofanelli M, Arnež ZM and Piccinato A: Prognostic indicators of improved survival and quality of life in surgically treated oral cancer. Oral Surg Oral Med Oral Pathol Oral Radiol. Jan 31, 2018 (Epub ahead of print). doi: 10.1016/j.0ooo.2018.01.016.

5. Blatt S, Krüger M, Ziebart T, Sagheb K, Schiegnitz E, Goetze E, Al-Nawas B and Pabst AM: Biomarkers in diagnosis and therapy of oral squamous cell carcinoma: A review of the literature. J Craniomaxillofac Surg 45: 722-730, 2017.

6. Omar E: Current concepts and future of noninvasive procedures for diagnosing oral squamous cell carcinoma-a systematic review. Head Face Med 11: 6, 2015.

7. Osaka R, Hayashi K, Onda T, Shibahara T and Matsuzaka K: Evaluation of liquid based cytology for tongue squamous cell carcinoma: Comparison with conventional cytology. Bull Tokyo Dent Coll 60: 29-37, 2019.

8. Kujan O, Huang G, Ravindran A, Vijayan M and Farah CS: CDK4, CDK6, cyclin D1 and Notch1 immunocytochemical expression of oral brush liquid-based cytology for the diagnosis of oral leukoplakia and oral cancer. J Oral Pathol Med 48: 566-573, 2019.

9. Sivakumar N, Narwal A, Kumar S, Kamboj M, Devi A, Pandiar D and Bhardwaj R: Application of the Bethesda system of reporting for cervical cytology to evaluate human papilloma virus induced changes in oral leukoplakia, oral squamous cell carcinoma, and oropharyngeal squamous cell carcinoma: A cytomorphological and genetic study. Diagn Cytopathol 49: 1036-1044, 2021.

10. Rossi ED, Bizzarro T, Schmitt F and Longatto-Filho A: The role of liquid-based cytology and ancillary techniques in pleural and pericardic effusions: An institutional experience. Cancer Cytopathol 123: 258-266, 2015.

11. Mehrotra R: The role of cytology in oral lesions: A review of recent improvements. Diagn Cytopathol 40: 73-83, 2012.

12. Tanuma JI, Shisa H, Hiai H, Higashi S, Yamada Y, Kamoto T, Hirayama Y, Matsuuchi $\mathrm{H}$ and Kitano M: Quantitative trait loci affecting 4-nitroquinoline 1-oxide-induced tongue carcinogenesis in the rat. Cancer Res 58: 1660-1664, 1998.

13. Tanuma JI, Kitano M, Shisa H and Hiai H: Polygenetic susceptibility and resistance to 4-nitroquinoline 1-oxide-induced tongue carcinomas in the rat. J Exp Anim Sci 41: 68-77, 2000.

14. Tanuma JI, Hiai H, Shisa H, Hirano M, Semba I, Nagaoka S and Kitano M: Carcinogenesis modifier loci in rat tongue are subject to frequent loss of heterozygosity. Int J Cancer 102: 638-642, 2002.

15. Tanuma JI, Fujii K, Hirano M, Matsuuchi H, Shisa H, Hiai H and Kitano M: Five quantitative trait loci affecting 4-nitroquinoline 1-oxide-induced tongue cancer in the rat. Jpn J Cancer Res 92: 610-616, 2001.

16. Suwa H, Hirano M, Kawarada K, Nagayama M, Ehara M, Muraki T, Shisa H, Sugiyama A, Sugimoto M, Hiai $\mathrm{H}$, et al: Pthlh, a promising cancer modifier gene in rat tongue carcinogenesis. Oncol Rep 31: 3-12, 2014.

17. Zuber J, Shi J, Wang E, Rappaport AR, Herrmann H, Sison EA, Magoon D, Qi J, Blatt K, Wunderlich M, et al: RNAi screen identifies Brd4 as a therapeutic target in acute myeloid leukaemia. Nature 478: 524-528, 2011.

18. Wu Y, Wang Y, Diao P, Zhang W, Li J, Ge H, Song Y, Li Z, Wang D, Liu L, et al: Therapeutic targeting of BRD4 in head neck squamous cell carcinoma. Theranostics 9: 1777-1793, 2019.

19. Lovén J, Hoke HA, Lin CY, Lau A, Orlando DA, Vakoc CR, Bradner JE, Lee TI and Young RA: Selective inhibition of tumor oncogenes by disruption of super-enhancers. Cell 153: 320-334, 2013.

20. Liu X, Li Q, Huang P, Tong D, Wu H and Zhang F: EGFR-mediated signaling pathway influences the sensitivity of oral squamous cell carcinoma to JQ1. J Cell Biochem 119: 8368-8377, 2018.

21. Zhao L, Li P, Zhao L, Wang M, Tong D, Meng Z, Zhang Q, Li Q and Zhang F: Expression and clinical value of PD-L1 which is regulated by BRD4 in tongue squamous cell carcinoma. J Cell Biochem 121: 1855-1869, 2020.

22. Cho HY, Lee SW, Jeon YH, Lee DH, Kim GW, Yoo J, Kim SY and Kwon SH: Combination of ACY-241 and JQ1 synergistically suppresses metastasis of HNSCC via regulation of MMP-2 and MMP-9. Int J Mol Sci 21: 6873, 2020.

23. Yamamoto T, Hirosue A, Nakamoto M, Yoshida R, Sakata J, Matsuoka Y, Kawahara K, Nagao Y, Nagata M, Takahashi N, et al: BRD4 promotes metastatic potential in oral squamous cell carcinoma through the epigenetic regulation of the MMP2 gene. $\mathrm{Br}$ J Cancer 123: 580-590, 2020 
24. Delmore JE, Issa GC, Lemieux ME, Rahl PB, Shi J, Jacobs HM, Kastritis E, Gilpatrick T, Paranal RM, Chesi M, et al: BET bromodomain inhibition as a therapeutic strategy to target c-Myc. Cell 146: 904-917, 2011.

25. Poter JR, Fisher BE, Baranello L, Liu JC, Kambach DM, Nie Z, Koh WS, Luo J, Stommel JM, Levens D and Batchelor E: Global inhibition with specific activation: How p53 and MYC redistribute the transcriptome in the DNA double-strand break response. Mol Cell 67: 1013-1025, 2017.

26. Ries JC, Schreiner D, Steininger H and Girod SC: p53 mutation and detection of p53 protein expression in oral leukoplakia and oral squamous cell carcinoma. Anticancer Res 18: 2031-2036, 1998.

27. Pallavi N, Nalabolu GRK and Hiremath SKS: Bcl-2 and c-Myc expression in oral dysplasia and oral squamous cell carcinoma: An immunohistochemical study to assess tumor progression. J Oral Maxillofac Pathol 22: 325-331, 2018.

28. Pérez-Sayáns M, Suárez-Peñaranda JM, Pilar G, Barros-Angueira F, Gándara-Rey JM and García-garcía A: What real influence does the proto-oncogene c-myc have in OSCC behavior? Oral Oncol 47: 688-692, 2011.

29. Papakosta V, Vairaktaris E, Vylliotis A, Derka S, Nkenke E, Vassiliou S, Lazaris A, Mourouzis C, Rallis G, Spyridonidou S, et al: The co-expression of c-myc and p53 increases and reaches a plateau early in oral oncogenesis. Anticancer Res 26: 2957-2962, 2006.

30. Norimatsu Y, Yamaguchi T, Taira T, Abe H, Sakamoto H Takenaka M, Yanoh K, Yoshinobu M, Irino S, Hirai Y and Kobayashi TK: Inter-observer reproducibility of endometrial cytology by the osaki study group method: utilising the becton dickinson surepath ${ }^{\mathrm{TM}}$ liquid-based cytology. Cytopathology 27: 472-478, 2016

31. Suzuki T, Isaka E, Hiraga C, Akiyama Y, Okamura M, Oomura Y, Hashimoto K, Sato K, Tanaka Y and Nomura T: New oral cytodiagnostic criteria predict change to oral epithelial dysplasia (OED) and cancerization. Oral Sci Int 18: 203-208, 2021.

32. Ogawa K, Tanuma JI, Hirano M, Hirayama Y, Semba I, Shisa H and Kitano M: Selective loss of resistant alleles at p15INK4B and p16INK4A genes in chemically-induced rat tongue cancers. Oral Oncol 42: 710-717, 2006.

33. Livak KJ and Schmittgen TD: Analysis of relative gene expression data using real-time quantitative PCR and the 2(-Delta Delta C(T)) method. Methods 25: 402-408, 2001.

34. Robin X, Turck N, Hainard A, Tiberti N, Lisacek F, Sanchez JC and Müller M: pROC: An open-source package for R and S+ to analyze and compare ROC curves. BMC Bioinformatics 12: 77 , 2011.

35. Kitano M: Host genes controlling the susceptibility and resistance to squamous cell carcinoma of the tongue in a rat model Pathol Int 50: 353-362, 2000.

36. Kanojia D and Vaidya MM: 4-Nitroquinoline-1-oxide induced experimental oral carcinogenesis. Oral Oncol 42: 655-667, 2006

37. Moon SM, Ahn MY, Kwon SM, Kim SA, Ahn SG and Yoon JH: Homeobox C5 expression is associated with the progression of 4-nitroquinoline 1-oxide-induced rat tongue carcinogenesis. J Oral Pathol Med 41: 470-476, 2012.

38. Arduino PG, Surace A, Carbone M, Elia A, Massolini G, Gandolfo S and Broccoletti R: Outcome of oral dysplasia: A retrospective hospital-based study of 207 patients with a long follow-up. J Oral Pathol Med 38: 540-544, 2009.

39. Ikeda M, Shima K, Kondo T and Semba I: Atypical immunohistochemical patterns can complement the histopathological diagnosis of oral premalignant lesions. J Oral Biosci 62: 93-98, 2020 .

40. Noda Y, Kondo Y, Sakai M, Sato S and Kishino M: Galectin-1 is a useful marker for detecting neoplastic squamous cells in oral cytology smears. Hum Pathol 52: 101-109, 2016.

41. Osugi Y: p53 expression in various stages of 4-nitroquinoline 1 -oxide induced carcinoma in the rat tongue. J Osaka Dent Univ 30: 29-35, 1996

42. Li S, Zhang S and Chen J: c-Myc induced upregulation of long non-coding RNA SNHG16 enhances progression and carcinogenesis in oral squamous cell carcinoma. Cancer Gene Ther 26 400-410, 2019.
43. Ota K, Fujimori H, Ueda M, Jono H, Shinriki S, Ota T, Sueyoshi T, Taura M, Taguma A, Kai H, et al: Midkine expression is correlated with an adverse prognosis and is down-regulated by p53 in oral squamous cell carcinoma. Int J Oncol 37: 797-804, 2010.

44. Loke SL, Neckers LM, Schwab G and Jaffe ES: c-myc protein in normal tissue. Effects of fixation on its apparent subcellular distribution. Am J Pathol 131: 29-37, 1988.

45. Lee CM: Transport of c-MYC by Kinesin-1 for proteasomal degradation in the cytoplasm. Biochim Biophys Acta 1843: 2027-2036, 2014

46. Pérez-Sayáns M, Suárez-Peñaranda JM, Padín-Iruegas E, Gayoso-Diz P, Reis-De Almeida M, Barros-Angueira F, Gándara-Vila P, Blanco-Carrión A and García-García A: Quantitative determination of c-myc facilitates the assessment of prognosis of OSCC patients. Oncol Rep 31: 1677-1682, 2014.

47. Bouchard C, Dittrich O, Kiermaier A, Dohmann K, Menkel A, Eilers $M$ and Lüscher B: Regulation of cyclin D2 gene expression by the Myc/Max/Mad network: Myc-dependent TRRAP recruitment and histone acetylation at the cyclin D2 promoter. Genes Dev 15: 2042-2047, 2001

48. Tumbarello DA and Turner CE: Hic-5 contributes to epithelial-mesenchymal transformation through a RhoA/ROCK-dependent pathway. J Cell Physiol 211: 736-747, 2007.

49. Hermeking H, Rago C, Schuhmacher M, Li Q, Barrett JF, Obaya AJ, O'Connell BC, Mateyak MK, Tam W, Kohlhuber F, et al: Identification of CDK4 as a target of c-MYC. Proc Natl Acad Sci USA 97: 2229-2234, 2000.

50. Yap CS, Peterson AL, Castellani G, Sedivy JM and Neretti N: Kinetic profiling of the c-Myc transcriptome and bioinformatic analysis of repressed gene promoters. Cell Cycle 10: 2184-2196, 2011.

51. Claassen GF and Hann SR: A role for transcriptional repression of p21CIP1 by c-Myc in overcoming transforming growth factor $\beta$-induced cell-cycle arrest. Proc Natl Acad Sci USA 97: 9498-9503, 2000.

52. Acosta JC, Ferrándiz N, Bretones G, Torrano V, Blanco R, Richard C, O'Connell B, Sedivy J, Delgado MD and León J: Myc inhibits p27-induced erythroid differentiation of leukemia cells by repressing erythroid master genes without reversing p27-mediated cell cycle arrest. Mol Cell Biol 28: 7286-7295, 2008.

53. Waitzberg AF, Nonogaki S, Nishimoto IN, Kowalski LP, Miguel RE, Brentani RR and Brentani MM: Clinical significance of c-myc and p53 expression in head and neck squamous cell carcinomas. Cancer Detect Prev 28: 178-186, 2004.

54. Ribeiro DA, Kitakawa D, Aparecida M, Domingues C, Cabral LAG, Marques MEA and Salvadori DMF: Survivin and inducible nitric oxide synthase production during 4NQO-induced rat tongue carcinogenesis: A possible relationship. Exp Mol Pathol 83: 131-137, 2007.

55. Vlajnic T, Savic S, Barascud A, Baschiera B, Bihl M, Grilli B, Herzog M, Rebetez J and Bubendorf L: Detection of ROS1-positive non-small cell lung cancer on cytological specimens using immunocytochemistry. Cancer Cytopathol 126: 421-429, 2018.

56. Nakra T, Nambirajan A, Guleria P, Phulware RH and Jain D: Insulinoma-associated protein 1 is a robust nuclear immunostain for the diagnosis of small cell lung carcinoma in cytology smears. Cancer Cytopathol 127: 539-548, 2019.

57. Jain D, Nambirajan A, Borczuk A, Chen G, Minami Y, Moreira AL, Motoi N, Papotti M, Rekhtman N, Russell PA, et al: Immunocytochemistry for predictive biomarker testing in lung cancer cytology. Cancer Cytopathol 127: 325-339, 2019.

58. Metovic J, Righi L, Delsedime L, Volante M and Papotti M: Role of immunocytochemistry in the cytological diagnosis of pulmonary tumors. Acta Cytol 64: 16-29, 2020.

59. Tone K, Ohno S, Honda M, Notsu A, Sasaki K and Sugino T: Application of enhancer of zeste homolog 2 immunocytochemistry to bile cytology. Cancer Cytopathol 129: 612-621, 2021.

This work is licensed under a Creative Commons Attribution-NonCommercial-NoDerivatives 4.0 International (CC BY-NC-ND 4.0) License. 